\title{
À propos d'une sculpture remarquable acquise par le musée de Nouvelle-Calédonie
}

\section{Emmanuel Kasarhérou}

\section{(2) OpenEdition \\ 1 Journals}

\section{Édition électronique}

URL : http://journals.openedition.org/jso/6864

DOI : $10.4000 /$ jso.6864

ISSN : 1760-7256

\section{Éditeur}

Société des océanistes

\section{Édition imprimée}

Date de publication : 15 octobre 2013

Pagination : 149-152

ISBN : 978-2-85430-035-2

ISSN : 0300-953x

\section{Référence électronique}

Emmanuel Kasarhérou, «À propos d'une sculpture remarquable acquise par le musée de NouvelleCalédonie », Journal de la Société des Océanistes [En ligne], 136-137 | 2013, mis en ligne le 20 octobre 2013, consulté le 16 avril 2020. URL : http://journals.openedition.org/jso/6864 ; DOI : https://doi.org/ $10.4000 /$ jso. 6864 


\title{
À propos d'une sculpture remarquable acquise par le musée de Nouvelle-Calédonie
}

par

\author{
Emmanuel KASARHÉROU ${ }^{*}$
}

Le 17 novembre 2012, le musée de NouvelleCalédonie a acquis en salle des ventes, à Chinon (Indre-et-Loire), une sculpture de $71 \mathrm{~cm}$ représentant un personnage barbu coiffé d'un tidi, présentée dans le catalogue de la vente ainsi :

«Figure androgyne assez finement travaillée [...] au beau visage "assyrien". Bois teinté de noir et rouge. "

Une grande étiquette collée sur la pièce indiquait :

«Divinité Tutélaire: Enlevée par l'expédition de Gatope le 10 septembre 1865 par Mr Eugène Leffet mon neveu, Enseigne de Vaisseau à bord du Fulton sur la case de Gondu et Poindi Pachili, Chef de la tribu sauvage et anthropophage de la Côte Nord-Ouest de la Nouvelle-Calédonie dans l'Océanie. Statuette qu'il a bien voulu me donner comme un gage de son affection à son arrivée à Saumur le 10 avril 1866, [signature] Braz [ou Braza ?]. »

Depuis 146 ans, aux dires du commissairepriseur, la sculpture était restée dans une grande demeure familiale de Saumur où il a été appelé pour procéder à la vente.

Natif de Saumur, Eugène Leffet (1838-1909) avait été officier de Marine puis avait embrassé une carrière politique. Les opérations de répression, auxquelles, en tant que jeune officier, il participa en septembre 1865 dans la région de VohKoné, avaient été ordonnées par le gouverneur Charles Guillain en représailles à l'assassinat en juillet 1865 dans la baie de Chasseloup (Voh) de l'équipage du caboteur La Reine-des-îles et de celui du cotre Le Secret en août 1865. Le chef Gon-

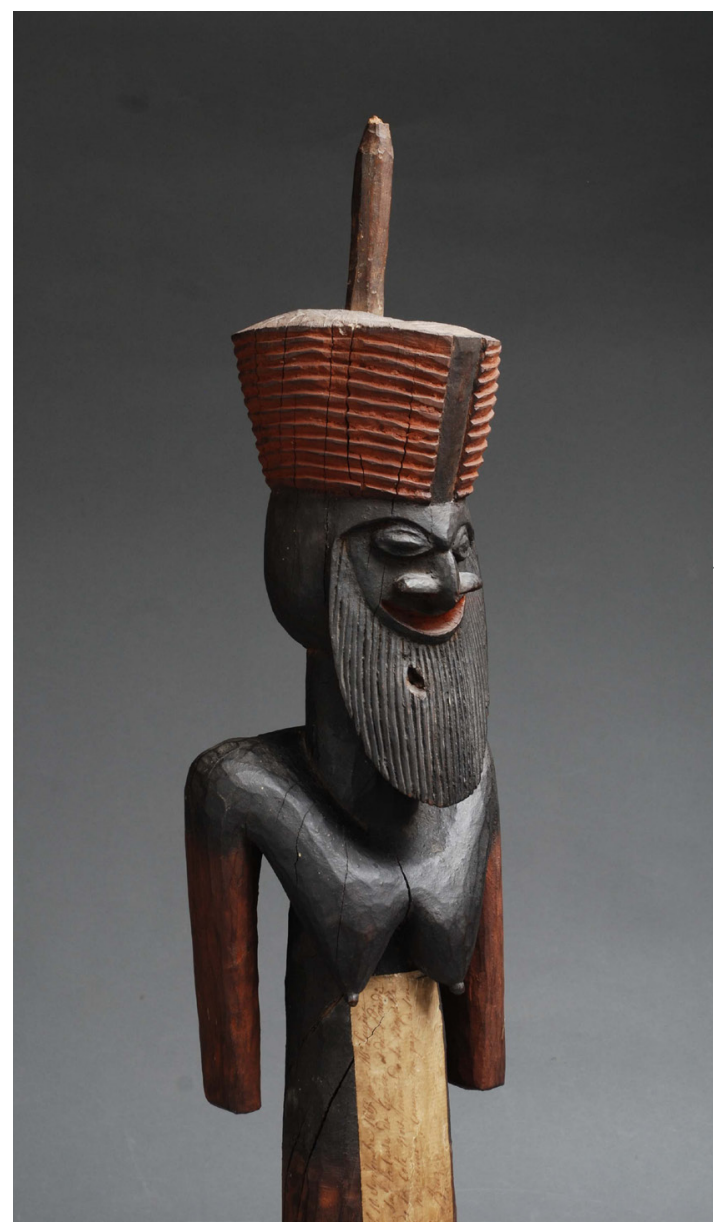

Рното 1. - Détail de la tête de la sculpture MNC 2012.21.1 (@ Merbelin, 2012) 


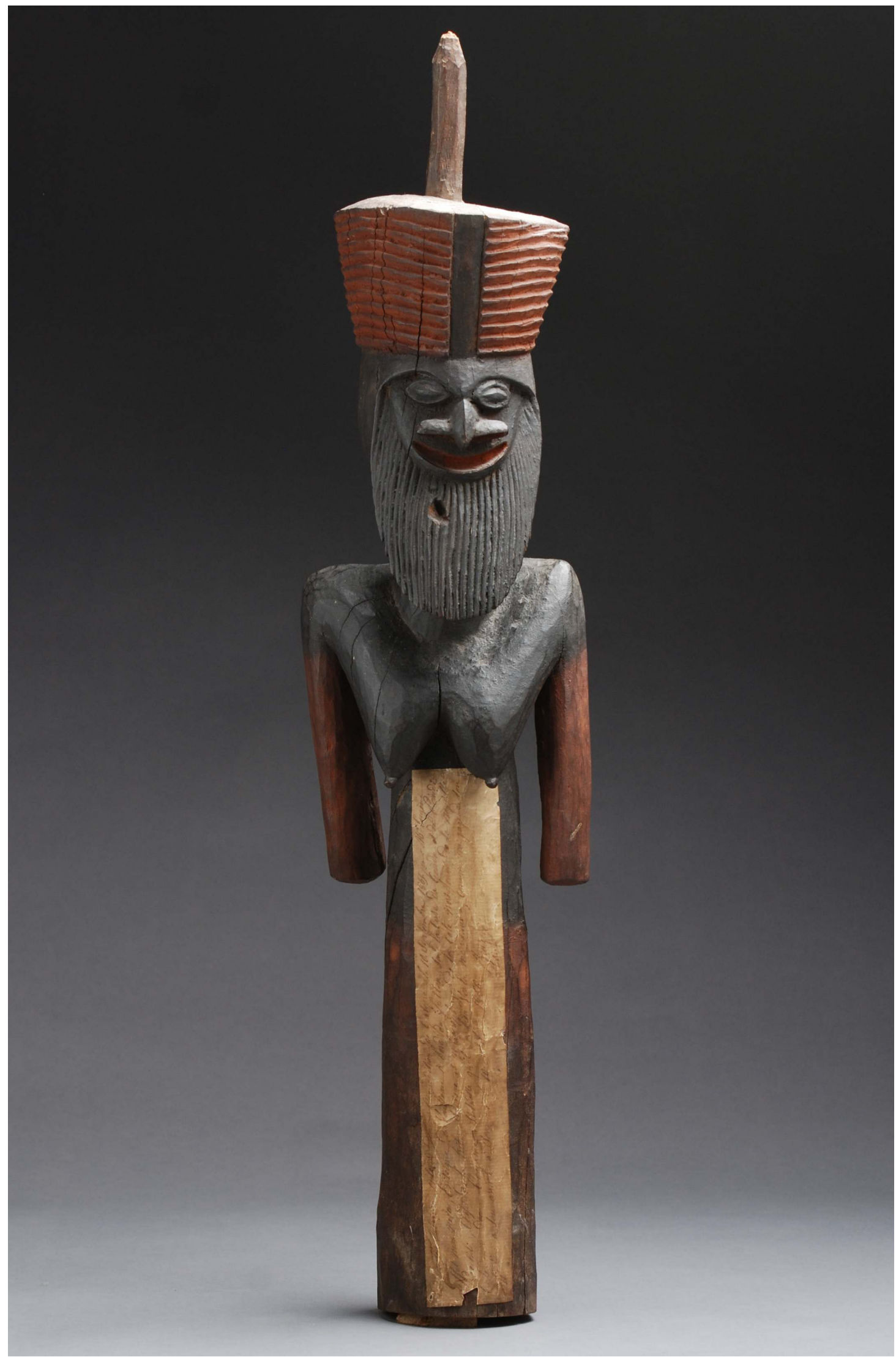

Рното 2. - Statuette de Gatope, prise par l'enseigne de vaisseau du Fulton, Eugène Leffet, «sur la case de Gondu... » et acquise récemment par le musée de Nouvelle-Calédonie MNC 2012.21.1 (@) Me Herbelin, 2012) 


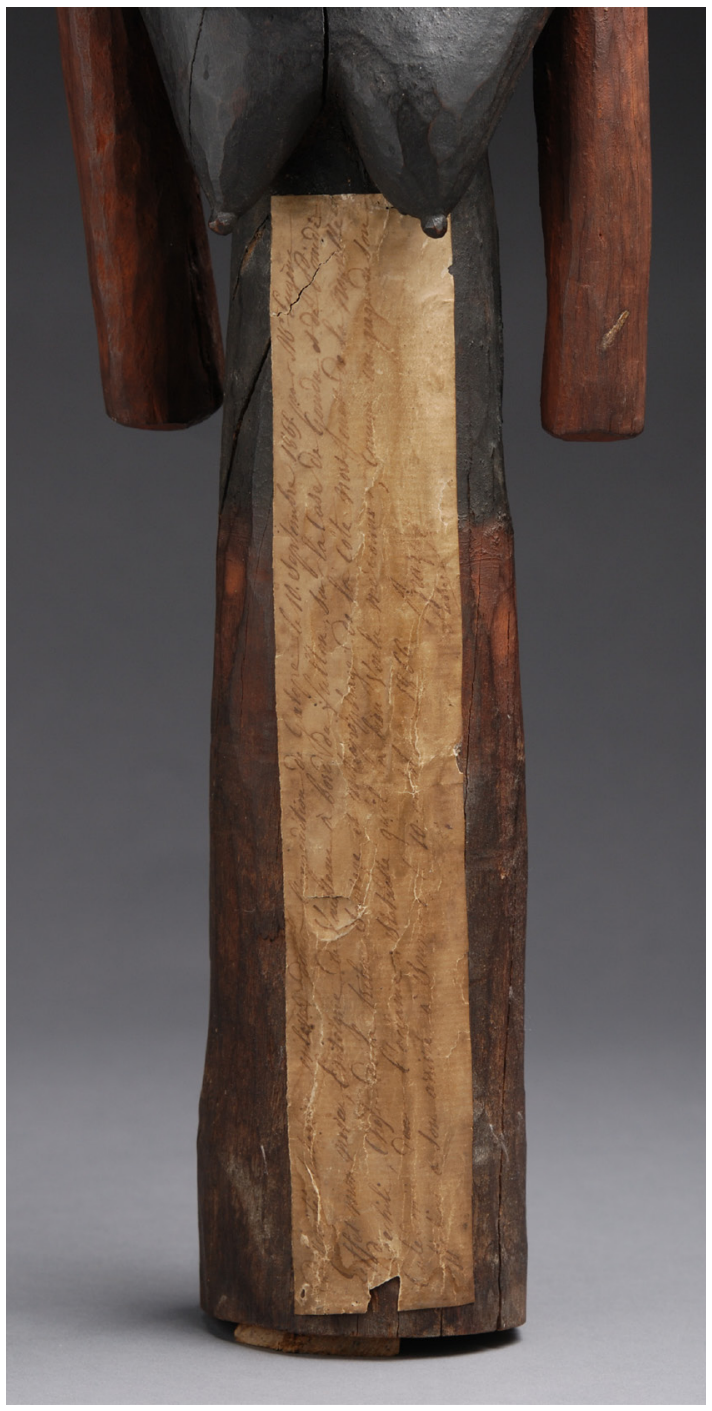

Рното 3. - Gros plan de l'étiquette collée sur de devant de la sculpture MNC 2012.21.1 (C) Me Herbelin, 2012)

dou (ca 1819-1869) ou "Goodu, membre du lignage Pwêêja (clan Görötû), venu de la côte Est jusqu'à Koohnê où les Pwêêja lui donnèrent la chefferie $[. .$.$] et adopté par le clan Nädù [. .]$. (Bensa, 2005 : 104) était soupçonné d'en être sinon l'instigateur du moins l'inspirateur, du fait de la guérilla armée qu'il entretenait depuis les hauteurs de la Chaîne centrale contre l'occupation française et tous ceux qui ne s'y opposaient pas activement. Le navire de transport La Fine et l'aviso à vapeur Fulton, sur lequel Eugène Leffet se trouvait, débarquèrent les troupes à Gatope (Voh) le 6 septembre 1865. Simultanément, la goélette La Gazelle convoyait le reste des troupes à Wagap sur la côte Est où le colon Taillard avait été assassiné par un membre de la tribu de Poindi-Poitchili au mois de juillet de la même année. Pwêdi Pwacili, transcrit Poindi-Poithili ou Patchili, était un chef de Wagap. Il s'était opposé à la présence française et, en 1862, s'était réfugié dans la Chaîne centrale d'où il s'allia à Gondou, formant l'une des premières coalitions armées contre la colonisation française. Le chef Mango ou Mwanggu Tyaunyën de Baco (Koné), exilé à cette époque à Gatope par Gondou (Guiart, 1963: 136), apporta son soutien aux troupes débarquées sur la côte Ouest, tandis que les tribus de Wagap et de Hienghène fournirent plusieurs centaines de supplétifs aux troupes débarquées sur la côte Est. Les deux colonnes firent leur jonction à Koné, dévastant sur leur passage plantations et villages.

Jules Garnier, présent dans la région, participa, bien que civil, à ces opérations militaires. Il nous a laissé un témoignage précieux sur la prise d'un village fortifié relevant de Gondou dans la Chaîne centrale le 10 septembre 1865 :

" Le chef Gondou avait une de ses résidences principales au village de Toono ; nos guides nous y conduisirent et nous nous trouvâmes en présence d'un véritable monument; je n'en avais encore jamais vu de semblable chez aucune des tribus visitées; ce palais indiquait un certain degré artistique. C'était une case immense et entourée d'une palissade composée d'énormes troncs d'arbres juxtaposés; chacun de ces géants des forêts était sculpté de façon à représenter plus ou moins bien des corps d'hommes gigantesques, tous dans des positions bizarres et différentes; ces statues étaient colorées au moyen de sanguine, leurs têtes étaient couronnées d'une immense sphère de minces lianes tressées qui représentaient assez bien la laineuse chevelure des Kanaks. Cette maison était placée dans un bas-fond, et lorsque nous l'aperçûmes du sommet voisin nous nous arrêtâmes un instant, prenant ces statues pour une troupe de géants. Un fossé profond circulait devant cette barrière. Dans son enceinte étaient de grandes perches plantées en terre, au sommet desquelles grimaçaient de nombreuses têtes de mort. »(Garnier, 1868 : 50)

C'est très probablement de ce village que provient la pièce rapportée par Eugène Leffet, puisque la date inscrite sur l'étiquette et celle donnée par Garnier concordent.

La pression de la présence française s'accroissait en ces années 1860 . Elle suscita des réactions d'abord locales. La conduite des opérations militaires induisit probablement un rapport nouveau à la guerre, poussant à la formation de coalitions, comme celle de Gondou. Parallèlement la construction de places fortifiées a probablement été stimulée de même que les magies de guerreset de protection, matérialisées par l'abondante et nouvelle production de sculptures dont le récit de Garnier témoigne.

Plus petite que les sculptures décrites dans son récit, la pièce acquise par le musée de NouvelleCalédonie est remarquable à bien des égards et d'abord par ses proportions et la finesse de son exécution. Le pied qui permettait de la ficher en terre a été scié. Il est peu probable qu'il s'agisse d'une pièce destinée à l'intérieur de la case, 
comme pourrait le suggérer son exceptionnel état de conservation. Il s'sagit bien plutôt d'une sculpture à planter, fraîchement sculptée et fichée autour de la case de cette case fortifiée, sans doute récemment construite.

La barbe et les seins bien que très marqués ne suffisent pas à en faire un être androgyne, par ailleurs inconnu dans la mythologie kanak. On retrouve ce caractère dans d'autres exemplaires de la statuaire kanak, comme sur une pièce très similaire de la collection Beer du Linden $\mathrm{Mu}-$ seum de Stuttgart (inv. 118856). Lamorce de représentation des bras brutalement interrompue par l'absence d'avant-bras est également une caractéristique que l'on rencontre sur d'autres pièces du même genre comme la sculpture à planter du Linden Museum, celle du musée de Bâle Sarasin, celle du musée d'Angoulême (inv. 934.1213), celle du musée d'Ethnographie de Vienne (inv. 105456) ou encore la petite sculpture à planter du musée du quai Branly (inv. 72.1989.3.1) et d'autres pièces du musée de l'université de Philadelphie.

L'acquisition par le musée de NouvelleCalédonie de ce remarquable objet vient compléter son importante collection en lui adjoignant un spécimen dont l'origine et les circonstances historiques de collecte font une pièce de référence pour l'histoire de l'art kanak et l'histoire de la Nouvelle-Calédonie (MNC 2012.21.1).

\section{BIBLIOGRAPHIE}

Bensa Alban, 2005. Histoire d'une chefferie kanak (Nouvelle-Calédonie). Récits paicî traduits et présentés par Alban Bensa et Atéa Antoine Goromindo, Paris, Karthala.

Garnier Jules, 1868. Voyage à la NouvelleCalédonie par M. Jules Garnier, Ingénieur civil des mines, 1863-1866, textes et dessins inédits, in E. Charton, Le Tour du Monde, Nouveau Journal des Voyages, publié sous la direction de M. Edouard Charton et illustré, Paris, Librairie Hachette, pp. 1-64.

Guiart Jean, 1963. Structures de la chefferie en Mélanésie du sud, Paris, Institut d'Ethnologie.

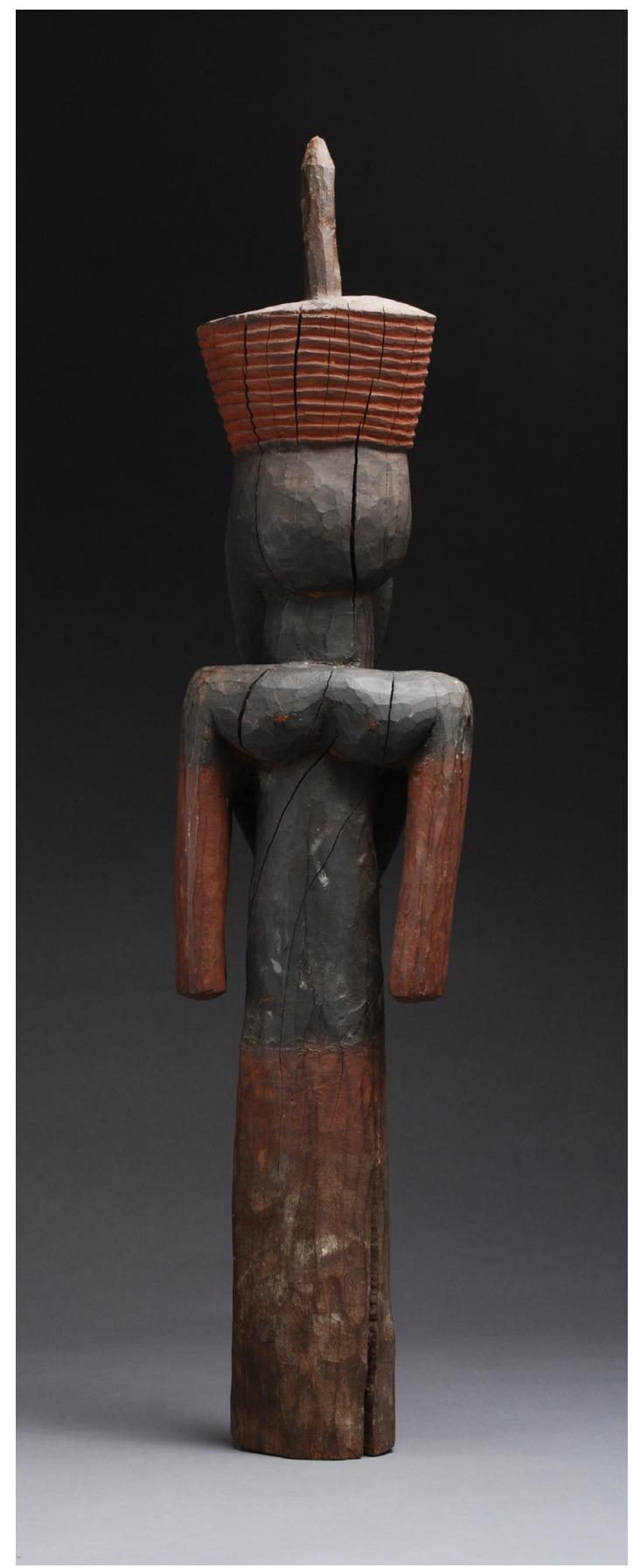

Рното 4. - Vue de dos de la sculpture MNC 2012.21.1 (C) Me Herbelin, 2012) 